\title{
Computed Tomography Assessment of Intestinal Gas Volumes in Functional Gastrointestinal Disorders
}

\author{
Sebastian R Mc Williams, ${ }^{1}$ Patrick D Mc Laughlin, ${ }^{1}$ Owen J O'Connor, ${ }^{1}$ Alan N Desmond, ${ }^{2,3}$ Áine Ní Laoíre, ${ }^{2}$ Fergus Shanahan, \\ Eamonn MM Quigley ${ }^{2,3}$ and Michael M Maher ${ }^{1,2,3 *}$ \\ Departments of ${ }^{1}$ Radiology and ${ }^{2}$ Medicine, and ${ }^{3}$ Alimentary Pharmabiotic Center, University College Cork and Cork University Hospital, Cork, \\ Ireland
}

\section{Background/Aims}

Many patients with functional gastrointestinal disorders (FGIDs) rank sensations of bloating and distension among their most debilitating symptoms. Previous studies that have examined intestinal gas volume (IGV) in patients with FGIDs have employed a variety of invasive and imaging techniques. These studies are limited by small numbers and have shown conflicting results. The aim of our study was to estimate, using CT of the abdomen and pelvis (CTAP), IGV in patients attending FGID clinic and to compare IGV in patients with and without FGID.

\section{Methods}

All CTAP $(n=312)$ performed on patients $(n=207)$ attending a specialized FGID clinic over 10-year period were included in this study. Patients were classified into one of 3 groups according to the established clinical grading system, as organic gastrointestinal disorder (OGID, ie, patients with an organic non-functional disorder, $n=84)$, FGID $(n=36)$ or organic and functional gastrointestinal disorder (OFGID, ie, patients with an organic and a functional disorder, $n=87$ ). Two independent readers blinded to the diagnostic group calculated IGV using threshold based 3D region growing with OsiriX.

Results

Median IGVs for the FGID, OGID, and OFGID groups were 197.6, 220.6 and $155.0 \mathrm{~mL}$, respectively. Stepwise linear regression revealed age at study, gender, and calculated body mass index to predict the log IGV with an $r^{2}$ of 0.116 , and $P<0.001$. There was a significant positive correlation between age and IGV in OGID (Spearman's $=0.253, P=0.02$ ) but this correlation was non-significant in the other groups.

\section{Conclusions}

Although bloating is a classic symptom in FGID patients, IGV may not be increased compared with OGID and OFGID patients.

(J Neurogastroenterol Motil 2012;18:419-425)

Key Words

Gastrointestinal diseases; Irritable bowel syndrome; Tomography, X-ray computed

Received: May 29, 2012 Revised: August 13, 2012 Accepted: August 22, 2012

(c) This is an Open Access article distributed under the terms of the Creative Commons Attribution Non-Commercial License (http://creativecommons. org/licenses/by-nc/3.0) which permits unrestricted non-commercial use, distribution, and reproduction in any medium, provided the original work is properly cited.

*Correspondence: Michael M Maher, MD, FRCR, FFR (RCSI)

Department of Radiology, Cork University Hospital, Wilton, Cork, Ireland

Tel: +353-21-4920279, Fax: +353-21-4920319, E-mail: m.maher@ucc.ie

Financial support: None.

Conflicts of interest: None. 


\section{Introduction}

Functional gastrointestinal disorders (FGIDs) including irritable bowel syndrome (IBS) represent a diverse group of disorders, characterized by gastrointestinal (GI) tract dysfunction in the absence of macro- or microscopic pathology. Bloating and distension feature prominently in the symptomatology of patients with IBS and other FGIDs and, in IBS, are typically associated with abdominal pain or discomfort and altered bowel habit. ${ }^{1-4}$ Despite the absence of evidence-based guidance in this regard, ${ }^{5}$ imaging is performed in many patients with FGIDs, particularly when there are symptoms or clinical or laboratory findings suggestive of underlying structural pathology. ${ }^{5}$ Computed tomography (CT) is increasingly performed because it is useful in identifying many of the organic pathologies, thought rightly or wrongly, which are at risk to be missed in this clinical scenario. Indeed, a recent study at our institution has shown that on presentation to specialist FGID clinic, CT of abdomen and pelvis (CTAP) was performed in approximately $11 \%$ of patients. Typically, these routine non-quantitative CTAPs were associated with low yield of positive imaging findings, and rarely identified plausible etiologies for clinical symptoms. ${ }^{6}$ The availability of CT images from such patients facilitated the current study which focused on investigating the feasibility and value of applying recently available image analysis software to the quantification of intestinal gas volumes (IGV) from clinically indicated CTAPs in FGID patients.

Though bloating and distension are prominent complaints among patients with IBS, the pathogenesis of these symptoms remains unclear. ${ }^{1}$ While impedance plethysmographic studies have demonstrated that the distension reported by patients is real and undergoes diurnal and meal-related fluctuations, ${ }^{7}$ studies on intra-abdominal or intestinal gas volumes have, in general, ${ }^{8-10}$ though not consistently, ${ }^{11,12}$ failed to describe any difference in these parameters between IBS and control subjects. In a series of elegant studies, the Barcelona group has demonstrated, in contrast, that the handling of intraluminal gas, as well as the diaphragmatic and abdominal wall musculature responses to gas, were impaired in IBS and gas transit. ${ }^{9,13-18}$ Among the discordant studies, there has been some studies that analyzed gas volumes on plain abdominal radiographs and $\mathrm{CT}$ scans and reported increased gas volumes in IBS. ${ }^{912-14}$

According to early studies, normal values for IGV range from $31-200 \mathrm{~mL} .{ }^{19}$ There have been a number of studies that in- vestigated IGV in patients with FGIDs using a variety of techniques and these have yielded conflicting results. The seminal work that investigated IGV in IBS patients, was done in the 1970s by Lasser et $\mathrm{al}^{10}$ who utilized a washout technique and showed IGV to be no different in IBS patients than in healthy controls. More recently, various in vivo techniques have been employed to directly and indirectly estimate IGV, including abdominal inductance plethysmography, ${ }^{20}$ radioisotope studies, ${ }^{15}$ plain film radiography ${ }^{11,12,21}$ and $\mathrm{CT}$ T $^{7-9,22}$

The handling of intestinal gas in patients with FGIDs is widely believed to be impaired. These patients' GI tracts have been shown to display a variety of defective or deficient homeostatic mechanisms for the normal transit of gas from mouth to anus. ${ }^{15-18}$ Knowledge of gas handling by the GI tract in FGIDs is based on empirical evidence. Firstly, when transit was pharmacologically decreased in the non-functionally impaired gut, symptoms arose akin to those in FGID patients. ${ }^{16}$ Decreased transit time was only part of the picture, however, as obstructed evacuation of gas was believed to cause more symptoms than the decreased transit. ${ }^{18}$ Abnormal retention was also a factor; by infusing gas into the jejunum, Serra et al. showed that patients with IBS retained gas more than healthy controls and this retention was associated with symptoms. ${ }^{17}$ In further study by Salvioli et al, ${ }^{15}$ scintigraphy was used to determine the location of retained gas in IBS patients. Gas was retained within the small intestine more often in IBS patients when compared to non-FGID controls. These results were congruent with Harder et $\mathrm{al}^{13}$ who demonstrated that jejunal gas infusion caused more symptoms than rectal infusion, in spite of the fact that absolute extent of abdominal distension was the same. The unifying mechanism causing symptoms in these patients is focal distension of the GI tract induced by gas. ${ }^{14}$ In FGIDs, the distribution of intestinal gas is believed to be an important determinant of patient's bloating symptom; the absolute IGV determines distension. In an early study on IBS patients by Maxton et al, ${ }^{7}$ the known diurnal variation in symptoms was not reflected in changes in IGV but rather by altered anteroposterior diameter of the abdomen.

It is tempting to use imaging for estimation or direct quantification of IGV in FGID patients as one may visualize intra-abdominal gas directly without relying on surrogate markers such as symptoms. Using plain abdominal radiography, a study by Chami et $\mathrm{al}^{11}$ suggested that IGV was increased in IBS patients compared with healthy controls. Koide et al ${ }^{12}$ developed a scoring system to grade IGV and showed the score to be increased in patients with IBS. However, a study by Morken et $\mathrm{al}^{21}$ yielded con- 
flicting results, suggesting that a gas score calculated from plain abdominal radiographs remained unchanged after lactulose challenge despite the pharmacologic challenge provoking symptoms in these IBS patients. The studies that employed plain radiography, therefore, are contradictory and are difficult to appraise, at least in part due to the methodological limitations; including small patient numbers, and potential for errors associated with projection radiography. Cross-sectional imaging studies and CT, in particular, would be much more accurate tools than plain radiography for accurate localization of intra-luminal gas, and the increasing availability of image analysis software with capability for segmentation based on Hounsfield unit value means that reproducible estimation of gas volumes is now feasible. ${ }^{23}$

We, therefore, sought to apply a new approach to the measurement of IGV by using CT image analysis and to address whether this widely available technology can detect differences in IGV between patients who were subsequently clinically diagnosed with organic, functional, or organic-functional GI disorders.

\section{Materials and Methods}

Institutional review board approval for this retrospective study was granted and the requirement for patient consent was waived. All patients $(\mathrm{N}=1,909)$ attending a specialist FGID clinic over a 10-year period (January 1999-January 2009) were selected and their diagnoses coded according to the International Classification of Disease (ICD-10) classification and the Rome III criteria for FGIDs. A subgroup of patients subsequently referred for CTAP during the study period $(\mathrm{n}=207$ ) was identified. Data concerning the indications for CTAP in individual patients were not available. All CTAPs $(n=312)$ performed on these patients were retrieved in DICOM format. Patients in this subgroup were divided into 3 groups based on clinical findings and classification according to clinical criteria as described above and as follows: organic GI disorders (OGID, $n$ $=84$ ), ie, patients with an organic non-functional disorder, FGID ( $\mathrm{n}=36$ ), ie, patients with a functional disorder but no organic disorder, and organic-functional GI disorders (OFGID, n $=87$ ), ie, patients with both organic and functional disorders. For the purpose of this study, the organic GI disorders were defined as all disorders of the GI tract including esophagus, stomach, liver, pancreas, small intestine and colon that are not explicitly defined in the Rome III criteria for functional gastrointestinal disorders. Examples include esophagitis, viral hepatitis, diverticulosis or colonic polyps. The proportion of each diagnosis in the 2 groups was compared with the Chi-squared test.

CTAPs ( $\mathrm{n}=312$ ) were acquired on one of 3 CT systems; a 64-slice multi-detector CT scanner (General Electric Lightspeed VCT-XTe; GE, Milwaukee, USA), 4-slice multi-detector CT scanner (Toshiba Aquilion II; Toshiba, Tochigi, Japan) or, in the case of older studies, a single slice CT scanner (Siemens Somatom; Siemens, Erlangen, Germany). The institutional CTAP protocol involves fasting for at least 6 hours prior to examination, administration of $100 \mathrm{~mL}$ of intravenous contrast and $1,000 \mathrm{~mL}$ of oral contrast (Gastrograffin, Bracco Diagnostics Inc., Princeton, $\mathrm{NJ}$, in $\mathrm{H}_{2} \mathrm{O}$ solution to give $2 \%$ dilution). All patients with prior bowel surgery, evidence of intestinal obstruction, or an abnormal gas collection related to perforation or abscess were excluded from the analysis. Only the most recent CTAP was considered for analysis when patients underwent more than one CTAP during the study period.

Two independent readers blinded to the diagnostic group calculated IGV using OsiriX (OsiriX Foundation, Geneva, Switzerland) with the 3-dimensional region-growing tool using lower and upper Hounsfield unit segmentation thresholds of -1024 $\mathrm{HU}$ and $-300 \mathrm{HU}$, respectively (Fig. 1). IGV was determined by estimating total gas volume in the abdominal cavity (gastrointestinal gas volume) and then subtracting the volume of gas in the stomach to yield IGV; differences between small and large intestinal gas were not calculated due to the possibility of confusing small and large intestinal gas. Calculation of IGV was done on

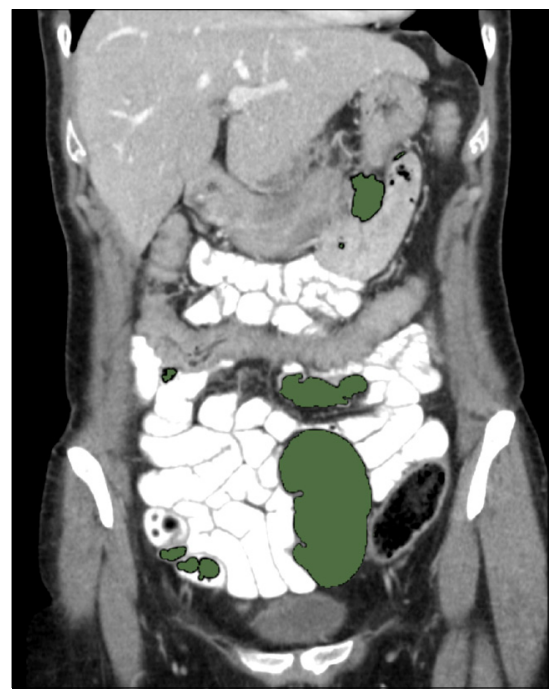

Figure 1. Intestinal gas volume estimation using $\mathrm{CT}$ images and threshold-based segmentation. 
image slices of $8 \mathrm{~mm}$ slice thickness, the same for all scanners. As the vast majority of IGV was in the form of several large pockets, scanner resolution difference that may have affected the sensitivity for small pockets was ignored. To control for body habitus which had previously been shown to correlate with IGV, the body mass index (BMI) was also retrospectively estimated from anthropometric measures of a single CTAP image at the L1 vertebral level according to published research (Fig. 2). ${ }^{24}$

All data were recorded using an Access 2007 database (Microsoft, Redmond, WA, USA) and statistical analyses were performed with SPSS version 14 (IBM, North Castle, NY, USA). For continuous variables, either ANOVA and Student's $t$ test or the Kruskall-Wallis and Mann-Whitney $U$ tests were used according to the normality of the sample distribution. Spearman correlations for age and IGV were performed. Simple linear regression with IGV as the dependent variable was performed to assess factors including BMI, gender, age at CT study and diagnostic subgroup (ie, FGID, OGID and OFGID). In addition, stepwise inclusion of factors was performed to identify those factors that most strongly impacted IGV.

\section{Results}

Significant differences in age were found between the diagnostic subgroups; patients in the FGID group were younger (mean 36.2, 56.6 and 46.7 years for FGID, OGID and OFGID, respectively; $P<0.001$ for all differences) but no statistically sig- nificant difference in BMI or gender was demonstrated between the diagnostic subgroups (Table 1). The 10 most common organic GI disorder diagnoses encountered in the OGID and OFGID groups are noted in Table 2. There was no significant difference in the proportion of the diagnoses between groups $(P=0.231)$.

The median gastrointestinal gas volumes were as follows: OGID, $283.7 \mathrm{~mL}$; FGID, $250.5 \mathrm{~mL}$; OFGID, $207.2 \mathrm{~mL}$. After removing the stomach volume, median IGVs were estimated as follows: $220.6 \mathrm{~mL}$ for OGID, $197.6 \mathrm{~mL}$ for FGID and $155.0 \mathrm{~mL}$ for OFGID, respectively. Mann-Whitney U-tests with Bonferroni correction revealed the difference in median IGV between OGID and OFGID was significantly different ( $P$ $=0.017)$ (Table 1). The differences between the OGID and FGID $(P=0.327)$ and FGID and OFGID $(P=0.291)$ were not significant. When comparing between diagnostic groups by gender, the difference in median IGV was only significant when comparing males in the OGID and OFGID group ( $P=$ $0.028)$. There also was a positive correlation between age and IGV for patients in the OGID subgroup only (Spearman's = $0.253, P=0.022)$.

Given the recognized diurnal variation in patients' symptoms in FGID, we also attempted to assess if the time at which CTAP was performed (morning vs afternoon) had any impact on IGV. Gas volumes estimated from CTAPs performed in the morning (before 12:00 pm) were compared with those estimated from CTAPs performed in the afternoon (after 12:00 pm). No significant difference in IGV was noted. Subgroup analysis showed
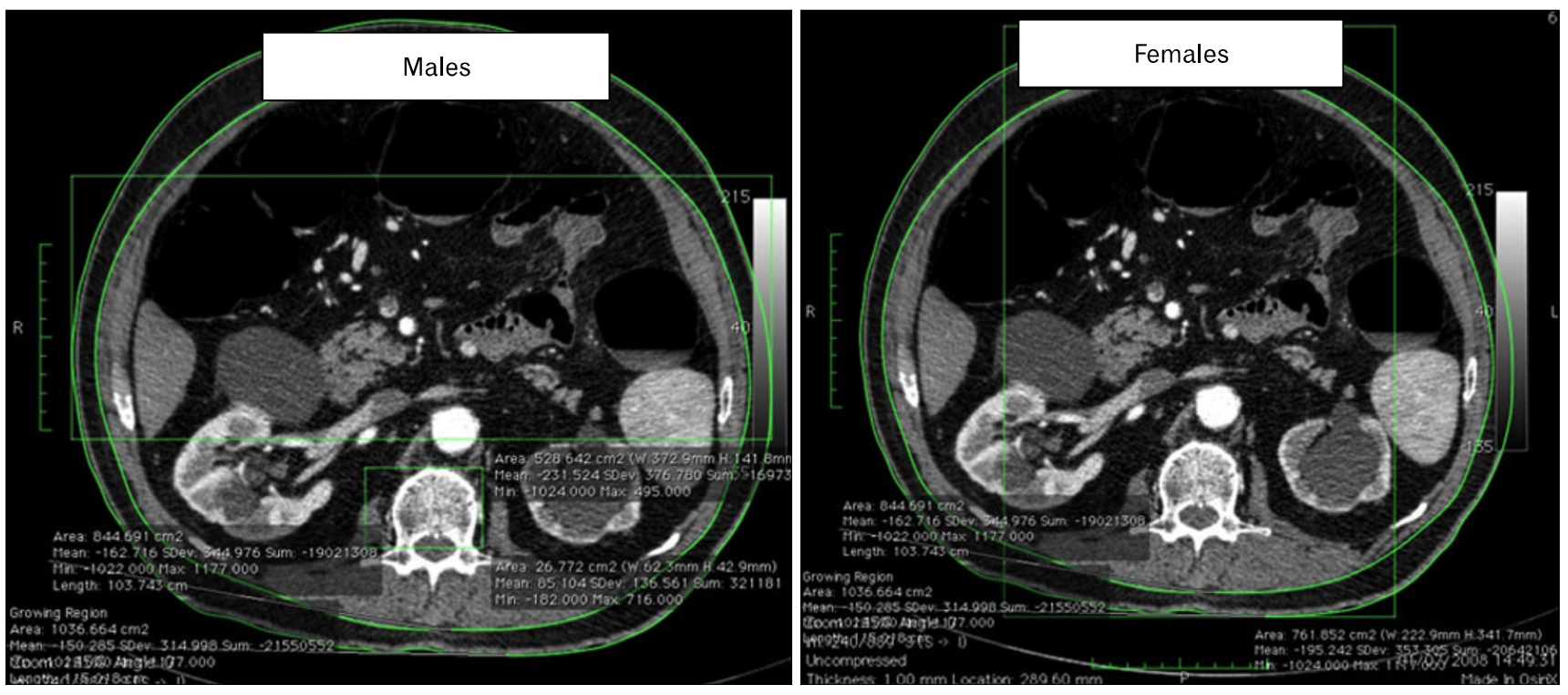

Figure 2. Anthropometric measures employed for calculating body mass index in male and female subjects. 
Table 1. Demographics and Intestinal Gas Volume Results by Diagnostic Group and Gender

\begin{tabular}{|c|c|c|c|c|c|c|c|c|c|c|}
\hline $\begin{array}{l}\text { Diagnostic } \\
\text { group }\end{array}$ & $\begin{array}{l}\text { Total } \\
\text { (n) }\end{array}$ & Gender & $\begin{array}{l}\text { By gender } \\
\text { (n) }\end{array}$ & $\begin{array}{c}\text { BMI } \\
(\text { mean } \pm \mathrm{SD} \\
\left.\mathrm{kg} / \mathrm{m}^{2}\right)\end{array}$ & $\begin{array}{l}\text { Median IGV } \\
\text { (IQR), mL }\end{array}$ & $\begin{array}{c}\text { Total median } \\
\text { IGV (IQR), } \\
\text { mL }\end{array}$ & $\begin{array}{l}\text { Pre-noon } \\
\text { (n) }\end{array}$ & $\begin{array}{c}\text { Pre-noon } \\
\text { median (IQR), } \\
\mathrm{mL}\end{array}$ & $\begin{array}{l}\text { Post-noon } \\
\text { (n) }\end{array}$ & $\begin{array}{c}\text { Post-noon } \\
\text { median IQR } \\
(\mathrm{IGV}), \mathrm{mL}\end{array}$ \\
\hline \multirow[t]{2}{*}{ FGID } & \multirow[t]{2}{*}{36} & & & $26.8 \pm 5.5$ & & \multirow[t]{2}{*}{$197.6(138.2)$} & & $187.7(202.4)$ & & $209.6(1$ \\
\hline & & Male & $10(18 \%)$ & $26.5 \pm 6.7$ & 184.6 & & 3 & $465.8^{\mathrm{a}}$ & 7 & 143.3 \\
\hline \multirow[t]{2}{*}{ OGID } & \multirow[t]{2}{*}{84} & Female & $48(57 \%)$ & $24.2 \pm 6.6$ & $170.9(204.0)^{b}$ & \multirow[t]{2}{*}{$220.6(279.3)^{c}$} & 9 & $149.5(281.7)$ & 39 & $173.8(198.3)$ \\
\hline & & & & $26.1 \pm 5.7$ & $304.3(231.2)^{b}$ & & 9 & & 27 & $334.6(209.5)$ \\
\hline \multirow[t]{2}{*}{ OFGID } & \multirow[t]{2}{*}{87} & Female & $55(63 \%)$ & $25.0 \pm 5.1$ & $150.7(145.1)$ & \multirow[t]{2}{*}{$155.0(170.0)^{c}$} & 12 & $116.6(108.2)$ & 43 & $154.4(181.9)$ \\
\hline & & Male & $32(37 \%)$ & $27.2 \pm 5.7$ & $180.6(187.8)$ & & 6 & $224.4(244.8)$ & 26 & $180.6(190.6)$ \\
\hline
\end{tabular}

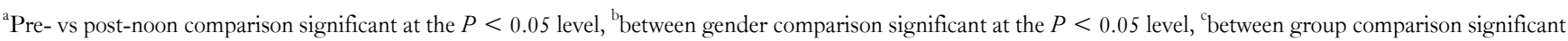
at the $P<0.05$ level.

BMI, body mass index; IGV, intestinal gas volume; IQR, interquartile range; FGID, functional gastrointestinal disorder; OGID, organic gastrointestinal disorder; OFGID, organic and functional gastrointestinal disorder.

Table 2. The 10 Most Common Organic Diagnoses in the Organic Gastrointestinal Disorder and Organic and Functional Gastrointestinal Disorder Groups

\begin{tabular}{lcc}
\hline \multicolumn{1}{c}{ Diagnosis } & OGID (n) & OFGID (n) \\
\hline GERD without esophagitis & 24 & 35 \\
Hiatus hernia & 21 & 24 \\
Gastritis/duodenitis & 20 & 21 \\
Helicobacter pylori infection & 19 & 20 \\
GERD with esophagitis & 17 & 21 \\
Peptic ulcer disease & 16 & 8 \\
Colonic diverticulosis & 12 & 22 \\
Colonic polyp & 11 & 12 \\
Crohn's disease & 10 & 5 \\
Barrett's esophagus & 8 & 14
\end{tabular}

OGID, organic gastrointestinal disorder; OFGID, organic and functional gastrointestinal disorder; GERD, gastroesophageal reflux disease.

There was no significant difference in the proportion of the diagnoses between groups $(P=0.231$, Chi-squared test).

a significant increase in IGV in a small number of male patients with FGIDs $(n=3)$ scanned before 12:00 pm compared with those scanned after 12:00 pm (Table 1). When IGV comparisons were limited to patients diagnosed with IBS, no significant difference was found between the different IBS diagnostic subgroups (ie, constipation predominant, diarrhea predominant or alternating).

As IGV was non-parametric, the linear regression model was computed with $\log _{\mathrm{IGV}}$ as the dependent variable. Factors included in the model were age at study, BMI, gender, diagnostic subgroup and time of CTAP. The linear regression model returned an $r^{2}$ of 0.119 suggesting $11.9 \%$ of the variation in $\log _{\text {IGV }}$ was explained by the model. A stepwise model of regression excluding diagnostic subgroup was then performed and revealed that age at study, gender and calculated BMI predicted the $\log _{I G V}$ with a similar $\mathrm{r}^{2}$ of 0.116 .

\section{Discussion}

In a recent $\mathrm{CT}$-based study investigating the change in distribution of gas during symptomatic periods, Accarino described caudo-ventral redistribution of abdominal contents in 47 patients with FGIDs. ${ }^{9}$ The authors noted diaphragmatic descent and anterior abdominal wall protrusion, yet there was no significant change in IGV, even when these patients were symptomatic. This study with relatively large patient numbers yielded interesting results, although confounding factors such as co-morbid organic disorders were not considered. The current study aimed to address the limitations of Accarino's study by estimating IGV in a large number of patients with suspected FGID, who were thoroughly evaluated clinically and characterized following established clinical scoring systems and then stratified into disease subgroups based on the above evaluation. Our primary aim, therefore, was to determine whether differences in IGV existed between subgroups of these patients who had been definitively characterized as having organic, functional, or organic-functional GI disorders.

Our study examined a total of 207 patients with suspected FGIDs and found a non-significant trend for lower and not greater IGV in patients who were ultimately diagnosed with FGIDs compared with those with OGIDs. Furthermore, IGVs were still and significantly lower than those with organic disease among those with concomitant functional and organic disorders, a finding that was not readily explained. It must be noted that the mean age was significantly different between the groups (56.6 
years for OGID, 36.2 years for FGID and 46.7 years for OFGID), an age distribution that reflects the typical demographic profile of patients with functional and organic gastrointestinal disorders, in general. Furthermore, we found that IGV significantly increased with advancing patient age in the OGID subgroup.

IGV is known to vary in a diurnal pattern with IGV being higher in the evening; this normal increase is known to correlate with an increase in reported symptoms in the evenings for patients with FGIDs. ${ }^{3}$ The only difference in IGV related to diurnal variation identified in our study was among males with FGIDs where a difference was noted between pre- and post-noon studies; however, this difference was likely an artifact explained by small numbers ( $\mathrm{n}=3$ for pre-noon group). Maxton et $\mathrm{al}^{7}$ considered the difference between pre- and post-noon studies in patients with IBS and also found no difference in IGV. Considering that patients underwent standard fasting and bowel prep, the expected rise in IGV may have been masked by factors on the preparation of CT study.

Accarino et $\mathrm{al}^{9}$ only considered constipation-predominant and alternating IBS in their analysis as they had observed the diarrhea-predominant to be less associated with bloating symptoms. Based on the results of current study, where no difference in IGV was observed between different IBS subtypes, it would appear that the bloating symptoms which were found more commonly in the constipation-predominant group in Accarino's paper may not be solely explainable by increased IGV.

An important aspect of this study is that it highlights that important additional objective data (eg, IGV) can be obtained from CT studies of the abdomen and pelvis, without modification of scanning protocol, which could be potentially useful in the future for advancing our understanding of IBS and other gastrointestinal disorders. These additional objective data may also aid in objective assessment of therapeutic regimens.

This study had several major limitations. First, as the patient population was an outpatient clinic population, only a small number of patients (approximately $11 \%$ of the total) were referred for CTAP. This constitutes selection bias as those referred may have had more complex, overlapping symptomatology or red-flag symptoms. Furthermore, data on the imaging requests were not available to evaluate specific indications for $\mathrm{CT}$ nor were data on the patients' symptoms at the time of CT.

In conclusion, we believe that the results of this study contribute to the limited body of knowledge surrounding the role of intestinal gas quantification in FGID patients. Quantitative CT evaluation of intestinal gas volume has not, to our knowledge, previously been reported in a group of patients referred for CT during the investigation of suspected FGID. The many variables which may theoretically confound our analysis such as the different severities of bloating at the time of CT, fasting status, medications and the younger age of FGID patients likely reflect the true spectrum of disease in patients referred to imaging departments worldwide during the investigation of FGIDs. In this cohort, we found no statistically significant difference in IGV between patients that were eventually diagnosed with FGIDs and those with OGIDs; therefore, routine quantitative CT analysis of intestinal gas appears not to contribute to patient care in this setting.

\section{Acknowledgements}

Authors' contribution: study design: SRM, OJO, AND, FS, EMMQ and MMM; manuscript drafting: SRM and PDM; data collection and analysis: SRM, AND and ANL; final manuscript supervision: FS, EMMQ and MMM.

\section{References}

1. Agrawal A, Whorwell PJ. Review article: abdominal bloating and distension in functional gastrointestinal disorders - epidemiology and exploration of possible mechanisms. Aliment Pharmacol Ther 2007; 27:2-10.

2. Azpiroz F, Malagelada JR. Abdominal bloating. Gastroenterology 2005;129:1060-1078.

3. Quigley EM. From comic relief to real understanding; how intestinal gas causes symptoms. Gut 2003;52:1659-1661.

4. Quigley EM. Impact of bloating and distention in irritable bowel syndrome: have we wandered too far from the manning creed? Clin Gastroenterol Hepatol 2009;7:7-8.

5. O'Connor OJ, McSweeney SE, McWilliams S, et al. Role of radiologic imaging in irritable bowel syndrome: evidence-based review. Radiology 2012;262:485-494.

6. Walsh K, McWilliams SR, Maher MM, Quigley EM. The spectrum of functional gastrointestinal disorders in a tertiary referral clinic in Ireland. Ir J Med Sci 2012;181:81-86.

7. Maxton DG, Martin DF, Whorwell PJ, Godfrey M. Abdominal distension in female patients with irritable bowel syndrome: exploration of possible mechanisms. Gut 1991;32:662-664.

8. Accarino A, Perez F, Azpiroz F, Quiroga S, Malagelada JR Intestinal gas and bloating: effect of prokinetic stimulation. Am J Gastroenterol 2008;103:2036-2042.

9. Accarino A, Perez F, Azpiroz F, Quiroga S, Malagelada JR. Abdominal distention results from caudo-ventral redistribution of contents. Gastroenterology 2009;136:1544-1551.

10. Lasser RB, Bond JH, Levitt MD. The role of intestinal gas in func- 
tional abdominal pain. N Engl J Med 1975;293:524-526.

11. Chami TN, Schuster MM, Bohlman ME, Pulliam TJ, Kamal N, Whitehead WE. A simple radiologic method to estimate the quantity of bowel gas. Am J Gastroenterol 1991;86:599-602.

12. Koide A, Yamaguchi T, Odaka T, et al. Quantitative analysis of bowel gas using plain abdominal radiograph in patients with irritable bowel syndrome. Am J Gastroenterol. 2000;95:1735-1741.

13. Harder H, Serra J, Azpiroz F, Passos MC, Aguadé S, Malagelada JR. Intestinal gas distribution determines abdominal symptoms. Gut 2003;52:1708-1713.

14. Malagelada JR. Sensation and gas dynamics in functional gastrointestinal disorders. Gut 2002;51(suppl 1):i72-i75.

15. Salvioli B, Serra J, Azpiroz F, et al. Origin of gas retention and symptoms in patients with bloating. Gastroenterology 2005;128:574-579.

16. Serra J, Azpiroz F, Malagelada JR. Intestinal gas dynamics and tolerance in humans. Gastroenterology 1998;115:542-550

17. Serra J, Azpiroz F, Malagelada JR. Impaired transit and tolerance of intestinal gas in the irritable bowel syndrome. Gut 2001;48:14-19.

18. Serra J, Azpiroz F, Malagelada JR. Mechanisms of intestinal gas retention in humans: impaired propulsion versus obstructed evacuation.
Am J Physiol Gastrointest Liver Physiol 2001;281:G138-G143.

19. Montalto M, Di Stefano M, Gasbarrini A, Corazza GR. Intestinal gas metabolism. Digest Liver Dis 2009;(suppl 3):27-29.

20. Lewis MJ, Reilly B, Houghton LA, Whorwell PJ. Ambulatory abdominal inductance plethysmography: towards objective assessment of abdominal distension in irritable bowel syndrome. Gut 2001;48: 216-220.

21. Morken MH, Berstad AE, Nysaeter G, Berstad A. Intestinal gas in plain abdominal radiographs does not correlate with symptoms after lactulose challenge. Eur J Gastroenterol Hepatol 2007;19:589-593.

22. Perez F, Accarino A, Azpiroz F, Quiroga S, Malagelada JR. Gas distribution within the human gut: effect of meals. Am J Gastroenterol 2007;102:842-849.

23. McWilliams SR, O'Connor OJ, McGarrigle AM, et al. CT-based estimation of intracavitary gas volumes using threshold-based segmentation: in vitro study to determine the optimal threshold range. J Med Imaging Radiat Oncol 2012;56:289-294.

24. Geraghty EM, Boone JM. Determination of height, weight, body mass index, and body surface area with a single abdominal CT image. Radiology 2003;228:857-863. 